\title{
Confining the Edges of the GW Vir Instability Strip
}

\author{
KLAUS WERNER, ${ }^{1}$ STEFAN DREIZLER, ${ }^{1}$ \\ ULRICH HEBER, ${ }^{2}$ AND THOMAS RAUCH ${ }^{1}$
}

\author{
${ }^{1}$ Institut für Astronomie und Astrophysik der Universität, 24098 Kiel, Germany \\ ${ }^{2}$ Dr. Remeis Sternwarte, Universität Erlangen-Nürnberg, 96049 Bamberg, Germany
}

We report on our NLTE model atmosphere analyses of PG 1159 stars. The results enable us to confine the location of the GW Vir instability region in the Hertzsprung-Russell diagram. The analysis of a spectrum of the non-pulsator PG $1520+525$ taken with the EUVE satellite in comparison with HST data of the pulsating protoype PG 1159-035 (=GW Vir) locates the blue edge of the instability strip near $T_{\text {eff }}=140000 \mathrm{~K}$ for stars in the respective luminosity range.

\section{The GW Vir Instability Strip}

PG 1159 stars are hydrogen-deficient and extremely hot pre-White Dwarfs. According to our current understanding they have lost their hydrogen envelope either by extraordinarily strong mass loss events or by ingestion and burning of hydrogen. This might be a consequence of a late thermal pulse during previous post-AGB evolution. Spectroscopically the PG 1159 stars are characterized by strong $\mathrm{C}$ IV and occasionally $\mathrm{O}$ VI lines, together with $\mathrm{He} \mathrm{II}$, but $\mathrm{H}$ Balmer lines are absent. Within the last years the number of known PG 1159 stars was brought up to 26. Eight stars of this group were identified as multimode nonradial g-mode pulsators (and four of the eight pulsators are Planetary Nebula central stars). They allow probing their interior structure by means of asteroseismology and thus play a key role in our understanding of post-AGB stellar evolution.

The GW Vir instability strip is defined by the pulsating PG 1159 stars. The pulsations are driven by cyclic ionization of $\mathrm{C}$ and $\mathrm{O}$ (Starrfield et al. 1984). The stellar pulsational model calculations can be subject to a stringent test if we compare the predicted instability region in the HRD with the observationally determined position of the PG 1159 stars.

\section{Spectroscopic Analyses}

Non-LTE model atmosphere analyses are in progress in order to find the photospheric parameters of all known PG 1159 stars. Figure 1 shows the positions of most of these objects in the $T_{\text {eff }}$-surface gravity plane. The models include $\mathrm{H}, \mathrm{He}, \mathrm{C}, \mathrm{O}$ and are computed with a NLTE code developed in Kiel and Bamberg. Most recent models include line blanketing by millions of lines from iron group elements. As an example Figure 2 shows the EUV flux of such an iron line blanketed model for a H-rich central star. More details on work done so far can be found in a recent review given by Dreizler, Werner \& Heber (1995).

Among the first PG 1159 stars analysed were the spectroscopic twins PG 1159-035 and PG $1520+525$. This pulsator/non-pulsator pair holds the key for the determination of the blue edge of the instability strip. Our optical analysis gave an equal $T_{\text {eff }}(140 \mathrm{kK})$ for both of them, however, within a large error range $( \pm 15 \mathrm{kK})$. The determination of more precise atmospheric parameters is hampered by the fact that temperature indicators in 


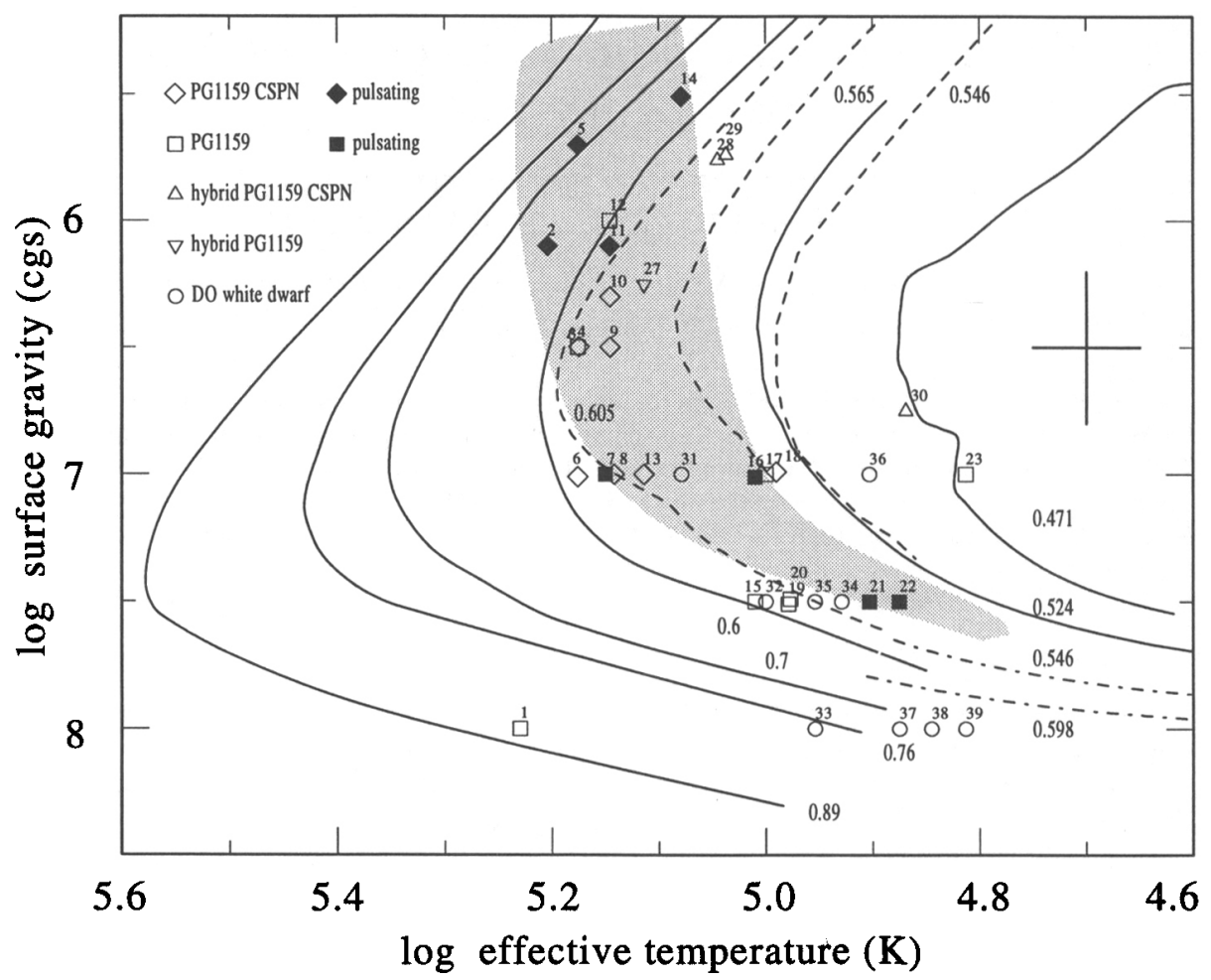

FIGURE 1. Location of the GW Vir instability strip (shaded region), comprising the pulsating PG 1159 stars (filled symbols). Evolutionary tracks are labeled with the respective stellar mass. A typical error bar is shown. For object identification see Table 1 (PG 1159 stars, labels 1-26) and Dreizler et al. (1995) for other objects

the optical spectral region are relatively insensitive. In the UV region, however, we can exploit the $\mathrm{O} v / \mathrm{O}$ VI ionisation equilibrium by line profile fitting. Alternatively, the EUV region can be used for line and continuum fitting. The latter is particularly useful because the PG 1159 stars have their flux maximum in the EUV range and the continuum flux and shape are very temperature sensitive. The oxygen ionization balance method has been used to derive a rather precise value for $T_{\text {eff }}$ of PG 1159-035 from a HST-FOS spectrum (Werner \& Heber 1993). The result was again $140 \mathrm{kK}$ but now with a much smaller uncertainty $( \pm 5 \mathrm{kK})$. No usable UV spectrum of the other twin was recorded up to now, so we decided to observe both twins with $E U V E$.

\section{EUVE Observation of PG $1520+525$}

Model atmospheres predict a very strong EUV flux, however, interstellar column densities are high. Therefore weak but still detectable fluxes at earth were expected in the region around $100 \AA$. Unfortunately the extinction towards PG 1159-035 turned out to be higher than expected: A 38ks exposure taken during Apr 06-07 (1993) is underexposed. On the other hand a 155ks exposure of PG 1520+525 taken on Feb 09-15 (1994) shows a noisy but still usable spectrum (Fig. 3). Comr ared to a $140 \mathrm{kK}$ model spectrum we 
central star NGC 1360 model flux at earth

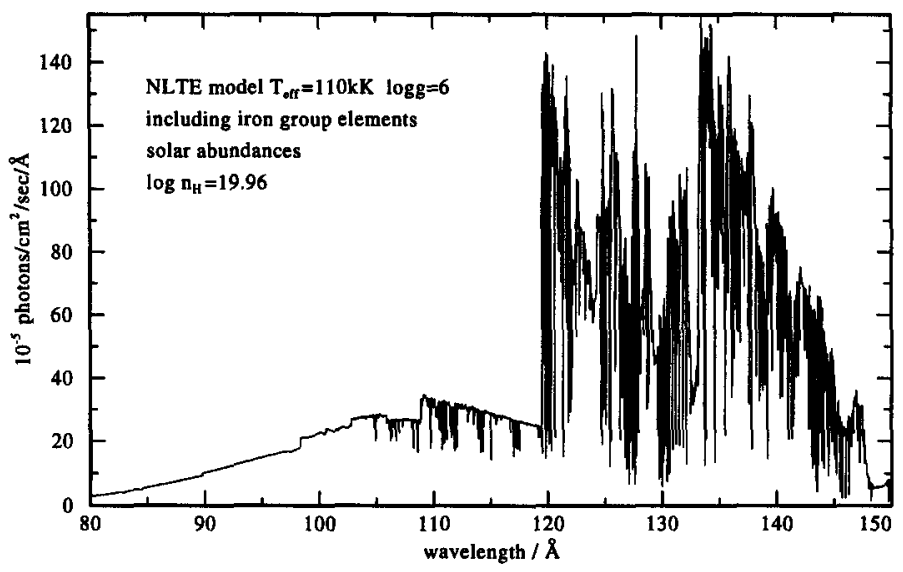

NGC 1360 predicted EUVE observation $t_{\text {exp }}=100 \mathrm{ks}$

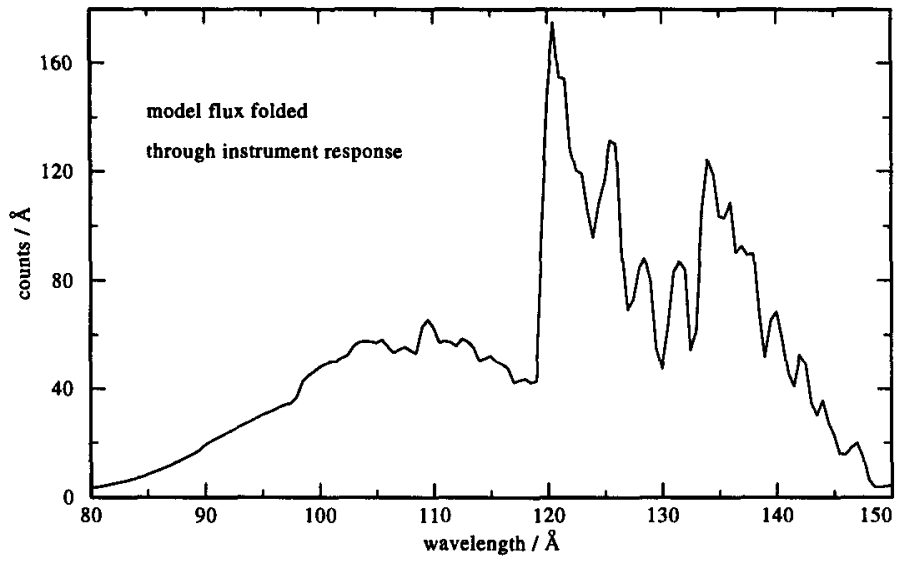

FigurE 2. Top: Non-LTE model flux for a hot planetary nebula central star. Line blanketing by iron group elements is included. Bottom: Model folded through the EUVE SW instrument response. Such models are currently under construction in order to analyze an EUVE spectrum of NGC 1360

would expect to see a strong $\mathrm{O} v$ absorption edge at $120 \AA$, but it is not detectable. We must have a higher $T_{\text {eff }}$ in order to depopulate $\mathrm{O} v$ and to weaken this edge: It can be seen that the $150 \mathrm{kK}$ model has a much weaker $\mathrm{O} v$ edge. We conclude that $T_{\text {eff }}$ of PG $1520+525(150 \mathrm{kK})$ is slightly higher than that of the pulsating prototype $(140 \mathrm{kK})$. Hence the blue edge of the GW Vir instability strip runs right between the loci of these two stars in the $T_{\text {eff }}-\log g$ plane.

Another pulsator/non-pulsator pair comprises PG 1707+427 and PG 1424+535. These define the red edge of the instability strip, which is found near $T_{\text {eff }}=100 \mathrm{kK}$. Analogously, this is what we learned from optical spectra. Their temperatures are much too low to produce enough EUV photons that could penetrate the ISM, so they cannot be detected 
PG $1520+525$ model fluxes at earth

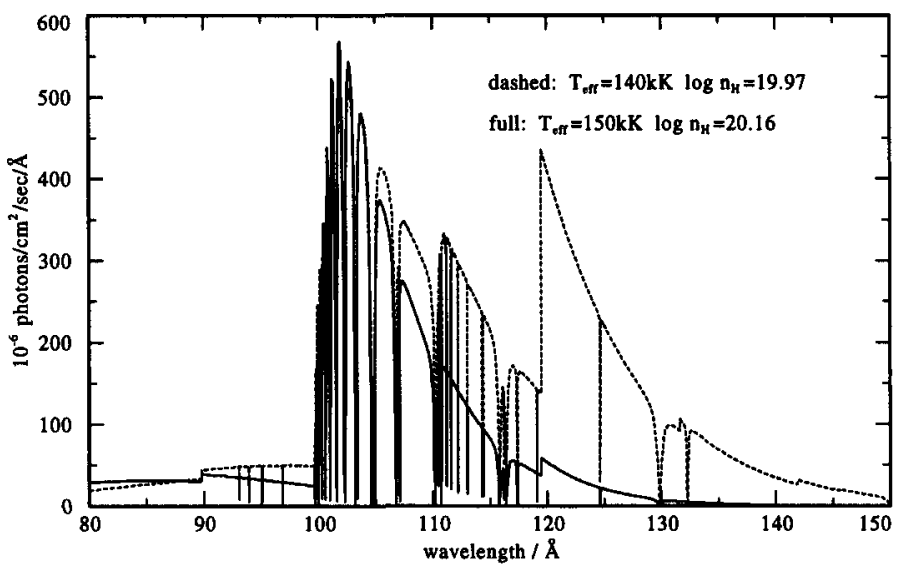

PG $1520+525$ EUVE observation $t_{\text {eap }}=155 \mathrm{ks}$

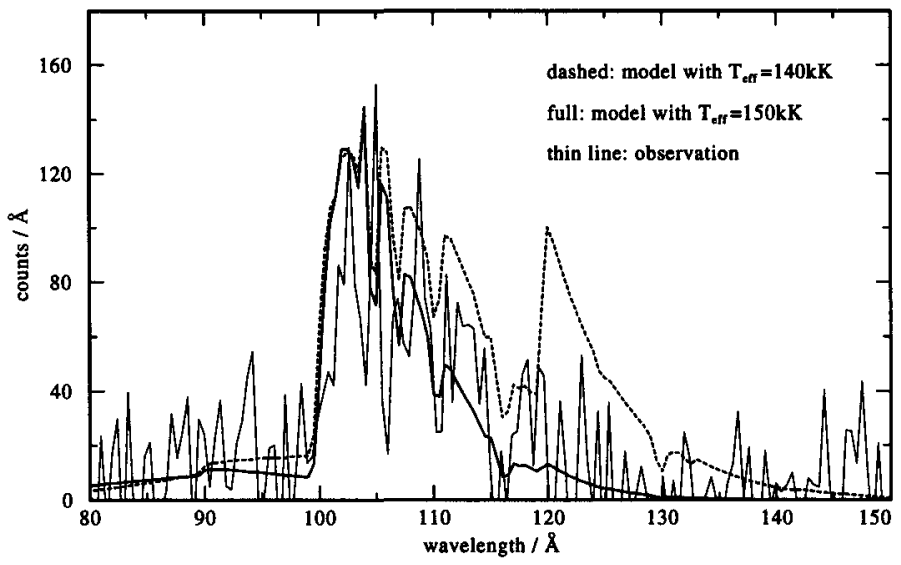

FigurE 3. Top: Non-LTE model fluxes (for two different effective temperatures) attenuated by the ISM. Note the strong $O \mathrm{~V}$ absorption edge at $120 \AA$ in the cooler model. It almost disappears when $T_{\text {eff }}$ is increased by only $10 \mathrm{kK}$. Another strong edge (at $100 \AA$ ) is due to O vI (2p). The absorption line spectrum is dominated by the respective $\mathrm{O}$ VI series as well as $\mathrm{C}$ IV lines. Other model parameters, taken from Werner et al. (1991): $\log g=7, \mathrm{He} / \mathrm{C} / \mathrm{O}=0.33 / 0.50 / 0.17$ (mass fractions).

Bottom: Both models folded through the EUVE SW instrument response and compared to the observation. The non-detection of the $120 \AA$ edge suggests that $T_{\text {eff }}$ exceeds $140000 \mathrm{~K}$

by EUVE. Instead, HST-GHRS observations are approved for Cycle 5 to find subtle differences in their atmospheric parameters.

After all, the picture as we have described it up to now is not as simple. The location of the instability region is not only dependent on surface gravity (and hence luminosity), but also on $\mathrm{C}$ and $\mathrm{O}$ abundances in the pulsation driving regions of the stars. Although these are very close below the photosphere, their composition may differ from the photospheric abundances. This fact might explain the presence of non-pulsators in the instability 
TABLE 1. The complete list of known PG 1159 stars (ordered by decreasing effective temperature), their spectroscopic subtype and atmospheric parameters $(:=$ uncertain). Columns 3 and 4 denote if the star is variable or has a planetary nebula. Abundances are given in \% mass fraction, $T_{\text {eff }}$ in $\mathrm{kK}$. The consecutive numbers in the last column refer to the labels in Fig. 1. For more details see Dreizler et al. (1995)

\begin{tabular}{|c|c|c|c|c|c|c|c|c|c|c|c|}
\hline Star & Type & Var. & PN & $T_{\text {eff }}$ & $\log g$ & $\mathrm{H}$ & $\mathrm{He}$ & $\mathrm{C}$ & $\mathrm{N}$ & $\mathrm{O}$ & $\mathrm{Nr}$ \\
\hline H $1504+65$ & Ep & no & no & 170 & 8.0 & & & 50 & & 50 & 1 \\
\hline $\mathrm{RXJ} 2117+3412$ & $\lg \mathrm{E}$ & yes & yes & 160 & 6.1 & & 38 & 56 & & 6 & \\
\hline PG 1144+005 & Ep & no & no & 150 & 6.5 & & 39 & 58 & 1.5 & 1.6 & \\
\hline Jn 1 & $\mathbf{E}$ & no & yes & 150: & 6.5 & & 30 & 46 & & 24 & \\
\hline NGC 246 & $\lg E$ & yes & yes & 150: & 5.7 & & 38 & 56 & & 6 & \\
\hline PG $1520+525$ & $\mathrm{E}$ & no & yes & 150 & 7.0 & $<8$ & 30 & 46 & $<0.3$ & 16 & \\
\hline PG 1159-035 & $\mathrm{E}$ & yes & no & 140 & 7.0 & $<8$ & 30 & 46 & $<0.3$ & 16 & \\
\hline NGC 650 & $\mathrm{E}$ & & yes & 140: & 7.0 & & & & & & \\
\hline Abell $21=\operatorname{Ym} 29$ & E & & yes & 140: & 6.5 & & 35 & 51 & & 14 & \\
\hline Longmore 3 & $\lg E$ & no & yes & 140: & 6.3 & & 38 & 56 & & 6 & 0 \\
\hline K 1-16 & $\lg E$ & yes & yes & 140: & 6.1 & & 38 & 56 & & 6 & \\
\hline PG 1151-029 & $\lg E$ & no & no & 140: & 6.0 & & 35 & 51 & & 14 & \\
\hline VV 47 & $\mathrm{E}$ & no & yes & 130: & 7.0 & & 35 & 51 & & 14 & \\
\hline Longmore 4 & $\lg E p$ & yes & yes & 120 & 5.5 & & 46 & 43 & & 11 & 4 \\
\hline HS $0444+0453$ & A & & no & 100: & 7.5 & & & & & & \\
\hline PG 1707+427 & A & yes & no & 100 & 7.0 & $<8$ & 30 & 46 & $<0.3$ & 16 & \\
\hline PG 1424+535 & A & no & no & 100 & 7.0 & $<8$ & 30 & 46 & $<0.3$ & 16 & \\
\hline IW & A & no & yes & 100: & 7.0 & & & & & & \\
\hline MCT $0130-1937$ & A & no & no & 95 & 7.5 & & 50: & 30 & & 20 & \\
\hline HS $1517+7403$ & A & & no & 95 & 7.5 & & 61 & 37 & & 2 & \\
\hline PG 2131+066 & A & yes & no & 80 & 7.5 & & 50: & 30 & & 20 & \\
\hline$P G 0122+200$ & A & yes & no & 75 & 7.5 & & 50 & 30 & & 20 & \\
\hline HS $0704+6153$ & A & & no & 65 & 7.0 & $<10$ & 44 & 26 & & 20 & \\
\hline NGC 6852 & $\lg E:$ & & yes & & & & & & & & \\
\hline NGC 6765 & $\lg E:$ & & yes & & & & & & & & \\
\hline Sh 2-78 & A & & yes & & & & & & & & \\
\hline
\end{tabular}

region. One example is HS $2324+3944$, a so-called hybrid-PG 1159 star (nr. 27 in Fig. 1). It does have hydrogen in the photosphere. If present in deeper layers, too, hydrogen would "poison" the pulsations.

K.W. acknowledges IAU and DFG (We 1312/7-1) travel grants and thanks Anne Miller (CEA) for support during $E U V E$ data reduction in Berkeley. This research is sponsored by the DFG (He 1356/16-2, We 1312/6-1) and the DARA (50 OR 94091).

\section{REFERENCES}

Dreizler, S., Werner, K. \& Heber, U. 1995, Analysis of PG 1159 stars and related objects, n White Dwarfs, ed. D. Koester \& K. Werner, Lect. Notes Phys., Springer, 443, 160

Starkfield, S., Cox, A. N., Kidman, R. B., \& Pesneld, W. D. 1984, Nonradial instability strips based on carbon and oxygen partial ionization in hot, evolved stars, ApJ, 281, 800

Werner, K. \& HeBer, U. 1993, UV spectroscopy of PG 1159 with HST and a prospective view of future EUVE observations, in White Dwarfs: Advances in Observation and Theory, ed. M. A. Barstow, Dordrecht: Kluwer, NATO ASI Series C, 403, 303 
Werner, K., Heber, U., Hunger, K. 1991, Non-LTE analysis of four PG1159 stars, A\&A, 244,437 